\title{
Calidad de Sueño en Médicos Residentes del HEODRA
}

\author{
Lilliam Mercedes Martínez', Indira Valeria Mayorga Coca', Neyling Vanessa Mendoza Hernández', Germán \\ Valdez Doña², Fannia Daniela Mairena López ${ }^{*}$
}

1. Estudiantes de IV año de Medicina, Facultad de Ciencias Médicas, Universidad Nacional Autónoma de Nicaragua, León (UNAN-León).

2. Departamento de Ciencias Fisiológicas, Facultad de Ciencias Médicas, Universidad Nacional Autónoma de Nicaragua, León (UNAN-León).

\section{RESUMEN}

La principal función fisiológica del sueño es mejorar el rendimiento físico y mental, esto ocurre si el sueño es de calidad y depende de diversos factores, El conocimiento de su calidad y factores que influyen en él y posibles trastornos, permitirán tener una base para buscar alternativas de prevención. El objetivo de esta investigación fue describir los factores que determinan un sueño de calidad en médicos residentes del Hospital Escuela Oscar Danilo Rosales. Fue descriptiva de corte trasversal realizada al $60 \%$ de médicos residentes de las especialidades. Se utilizó como instrumento de medición el índice de Calidad del Sueño de Pittsburg, proporciona una puntuación global de calidad y puntuaciones parciales en 7 componentes. Los factores incidentes en un sueño de calidad son laborales y el consumo de café, en todas las especialidades. Los principales trastornos del sueño que presentan los médicos residentes son: Insomnio de conciliación, insomnio de mantenimiento y sueño no reparador, dificultando la capacidad de concentración en la realización de las actividades asignadas. Se llegó a la conclusión que la calidad del sueño de los médicos residentes encuestados tiene un puntaje mayor a 5 puntos, considerado MALA CALIDAD DEL SUEÑO, la especialidad con mayor puntaje es Medicina interna.

Palabras claves: calidad de sueño, residentes, HEODRA, León.

\section{INTRODUCCIÓN}

El sueño se puede definir en términos sencillos como un estado reversible de desconexión sensorial y falta de respuesta al entorno. No ha sido fácil determinar las funciones que tiene el sueño y están lejos de que sean plenamente establecidas.

Sin embargo, con el advenimiento de la medicina del sueño y su interés en esta área, se han podido delinear una gran cantidad de trastornos y las enfermedades que los pueden desencadenar. Se conocen mejor los efectos de los trastornos del sueño sobre múltiples aspectos de la calidad de vida y el estado general de salud que funciones pueda tener el buen dormir. ${ }^{[1]}$

El ser humano duerme durante una tercera parte de su vida y de la calidad con que lo hace depende su desempeño en la vida cotidiana. La facilidad con que se logra entrar en sueño, la capacidad de mantener su continuidad y la sensación reparadora al día siguiente, son todos elementos que característicamente se alteran de una u otra forma en los individuos con un "mal" dormir.

Es decir, se demoran para conciliar el sueño, despiertan varias veces en la noche y al día siguiente se sienten cansados, somnolientos y su desempeño está disminuido. ${ }^{[2]}$
En condiciones normales el sueño está caracterizado por etapas superficiales y profundas que suceden cíclicamente durante toda la noche. El perfil de aparición de las distintas etapas de sueño es variable para cada persona.

Se conoce sin embargo, la progresión habitual en sujetos normales de estas etapas y está definida la proporción que ocupa cada una de ellas en el tiempo total de sueño que para que se cumpla se debe dormir por lo menos 8 horas. ${ }^{[2]}$

Existen muchos trabajos en los que no se puede cumplir estas 8 horas necesarias para empezar estas etapas y gozar de sus beneficios, como es el ser Médico, en el que se requiere estar despierto por más de 28 horas, sin períodos de descanso.

Es por eso que nos propusimos investigar la calidad del sueño en estos Médicos, especialmente los residentes de las distintas especialidades del Hospital Escuela Oscar Danilo Rosales, que además de sus responsabilidades como médicos tienen responsabilidades académicas, familiares entre otras.

Y cómo el no cumplir con las distintas etapas del ciclo del sueño interfiere en sus actividades laborales y en su vida social. 


\section{DISEÑO METODOLÓGICO}

Esta investigación fue de tipo descriptiva de corte transversal, se realizó a los médicos residentes de las diferentes especialidades de todos los años académicos del Hospital Escuela Oscar Danilo Rosales Argüello (HEODRA), con el objetivo de describir los principales factores que determinan un sueño de calidad en sus vidas, en relación a las actividades laborales y sociales que ejercen.

Universo: 80 médicos residentes de las especialidades de Ortopedia, Cirugía, Pediatría, Medicina Interna y Ginecología.

\section{Criterios de inclusión}

1. Residentes de Ortopedia, Cirugía, Pediatría, Medicina Interna y Ginecología, de I, II, III y IV año si corresponde.

\section{Laborar en el HEODRA.}

3. Realización de turnos por lo menos una vez a la semana.

\section{Criterios de exclusión}

1. Médico de base y de otra índole ajena a residencia de especialidad.

2. Residentes de subespecialidad.

3. No trabajador del HEODRA.

4. No realice turnos.

La fuente de información es primaria porque se entrevistó a la población de estudio directamente.

La recolección de información se realizó a través de un cuestionario, la que fue llenada por la propia población de estudio.

\section{Instrumento}

Se utilizó como instrumento de medición el índice de Calidad del Sueño de Pittsburg para evaluar la calidad del sueño, que proporciona una puntuación global de su calidad y puntuaciones parciales en siete componentes distintos: calidad subjetiva del sueño, latencia, eficiencia habitual, alteraciones, uso de medicación hipnótica y disfunción diurna.
Tabla 1. Mediana de la edad en años y sexo de los médicos residentes encuestados de las especialidades del HEODRA, Marzo a Octubre 2007

\begin{tabular}{lccccc}
\hline \multirow{2}{*}{ Especialidad } & \multirow{5}{*}{ Edad } & \multicolumn{5}{c}{ Sexo } \\
\cline { 3 - 6 } & & \multicolumn{2}{c}{ Femenino } & \multicolumn{3}{c}{ Masculino } \\
\cline { 3 - 6 } & $\mathrm{N}^{\circ}$ & $\%$ & $\mathrm{~N}^{\circ}$ & $\%$ \\
\hline Medicina Interna & 29 & 2 & 20 & 8 & 80 \\
Ortopedia & 29 & 3 & 27 & 8 & 73 \\
Pediatría & 30 & 2 & 18 & 9 & 82 \\
Cirugía & 28 & 2 & 17 & 10 & 83 \\
Ginecología & 29 & 10 & 71 & 4 & 29 \\
\hline Total & & 19 & 33 & 39 & 67 \\
\hline
\end{tabular}

Fuente Primaria

\section{RESULTADOS Y DISCUSIÓN}

La mediana de las edades de los encuestados que más predominó fue 29 años, lo que indica que estas son las edades en que más se afecta la calidad del sueño en los residentes, datos que se relacionan con lo referido por Aragón ${ }^{[1]}$ en su artículo "Alteraciones del sueño en psiquiatría" y el sexo de más predominio en las especialidades es el masculino por encima del $60 \%$ de los encuestados, excepto en Ginecología en donde el que más relevancia tiene es el femenino, por tanto la población que tiene mala calidad del sueño y alteraciones del sueño son en su mayoría los del sexo masculino, Aragón ${ }^{[1]}$ hace relación en base a las actividades que se asignan de acuerdo a cada sexo. (ver tabla 1)

La calidad del sueño con más afectaciones es la de los residentes de medicina interna con una mediana de 9 puntos, siguiéndole en orden de frecuencia los de pediatría y cirugía, siendo los de mejor calidad del sueño los de ginecología, estos resultados se asocian a las mayores responsabilidades y obligaciones que tienen los residentes de medicina interna y pediatría, situación que es reflejada en la práctica diaria. (ver figura 1)

Los factores de riesgo que se relacionan más con la afectación de la calidad del sueño son los laborales, lo que tiene afinidad con el número de responsabilidades y obligaciones asignadas, correlacionándose con la opinión de Miró[i] en su estudio "Influencia de la cantidad y calidad subjetiva del sueño en la ansiedad y el estado de ánimo deprimido" que manifiesta que los factores laborales son los más influyentes en la calidad del sueño. 
Figura 1. Medidas de tendencia central de la calidad del sueño de los médicos residentes encuestados de las especialidades del HEODRA, Marzo a Octubre 2007

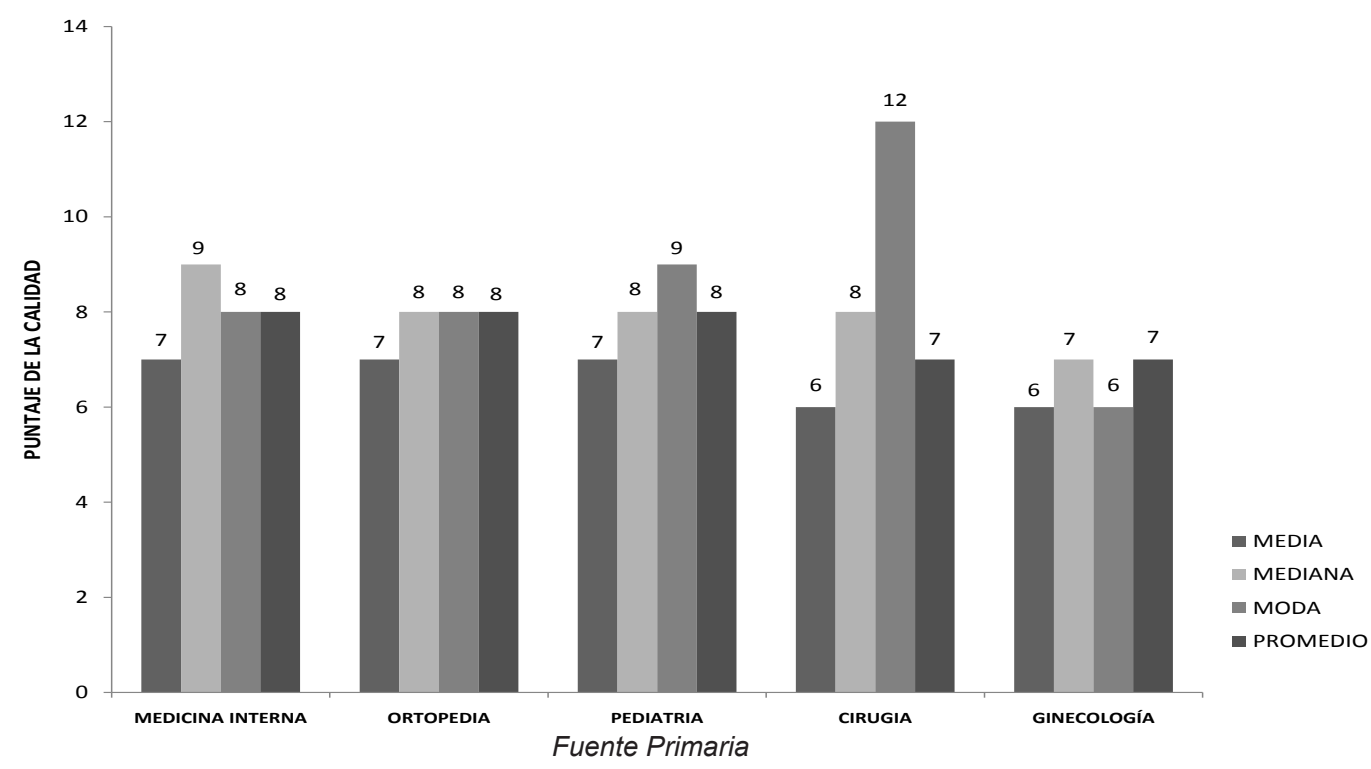

Tabla 2. Factores de riesgo que inciden en la calidad del sueño de los médicos residentes encuestados de las especialidades del HEODRA, Marzo a Octubre 2007

\begin{tabular}{|c|c|c|c|c|c|c|c|c|c|c|c|c|c|c|}
\hline \multirow[b]{2}{*}{ Especialidad } & \multicolumn{2}{|c|}{ Familiares } & \multicolumn{2}{|c|}{ Laborales } & \multicolumn{2}{|c|}{ Individuales } & \multicolumn{2}{|c|}{ Sociales } & \multicolumn{2}{|c|}{ Exámenes } & \multicolumn{2}{|c|}{ Café } & \multicolumn{2}{|c|}{ Tabaco } \\
\hline & № & $\%$ & $\mathrm{~N}^{\circ}$ & $\%$ & № & $\%$ & $\mathrm{~N}^{\circ}$ & $\%$ & № & $\%$ & № & $\%$ & № & $\%$ \\
\hline Medicina Interna & 6 & 60 & 9 & 90 & 2 & 20 & 2 & 20 & 5 & 50 & 7 & 70 & 1 & 10 \\
\hline Ortopedia & 5 & 45 & 8 & 73 & 4 & 36 & 3 & 27 & 5 & 45 & 8 & 73 & 0 & 0 \\
\hline Pediatría & 4 & 36 & 8 & 73 & 3 & 27 & 2 & 18 & 7 & 64 & 6 & 55 & 0 & 0 \\
\hline Cirugía & 1 & 8 & 6 & 50 & 2 & 17 & 2 & 17 & 6 & 50 & 7 & 58 & 0 & 0 \\
\hline Ginecología & 12 & 86 & 11 & 79 & 9 & 57 & 8 & 57 & 8 & 57 & 8 & 57 & 0 & 0 \\
\hline
\end{tabular}

Fuente Primaria

Tabla 3. Trastornos del sueño encontrados en los médicos residentes encuestados de las especialidades del HEODRA, Marzo a Octubre del 2007

\begin{tabular}{lccccc}
\hline Trastornos & Medicina Interna & Ortopedia & Cirugía & Ginecología & Pediatría \\
\hline Insomnio de conciliación & 3 & 3 & 2 & 4 & 6 \\
Insomnio de Mantenimiento & 4 & 0 & 3 & 0 & 7 \\
Insomnio tardío & 5 & 0 & 4 & 4 & 5 \\
Sueño no reparador & 6 & 3 & 5 & 4 & 4 \\
Insomnio transitorio & 3 & 4 & 2 & 0 & 0 \\
Insomnio de corta duración & 0 & 3 & 0 & 2 & 0 \\
Pesadillas & 0 & 2 & 0 & 4 & 0 \\
Somniloquia & 0 & 0 & 0 & 3 & 2 \\
Retraso de fase & 0 & 0 & 0 & 2 & 0 \\
\hline
\end{tabular}

Fuente Primaria 
Los residentes de las especialidades consumen en su mayoría café para mantenerse despiertos, lo que indica que este es otro de los factores de riesgo que inciden en el padecimiento de trastornos del sueño así como de una mala calidad de sueño. (ver tabla 2).

De los trastorno identificados en la población a estudio los de mayor frecuencia en las especialidades fueron trastornos de conciliación y sueño no reparador lo que indica que generalmente los residentes no logran llegar a la fase IV del sueño debido a que están pensando en las ocupaciones futuras que deben de realizar y esto no les permite dormir adecuadamente, explicación respaldada por Velluti ${ }^{[5]}$ en su esquema de la fisiología del sueño. (ver tabla 3 )

En relación al consumo de sustancias para conciliar el sueño en sus compañeros de trabajo solamente en las especialidades de medicina interna y ortopedia se encontró el consumo de Ansiolíticos como Alprazolam, que son otros de los factores que inciden en la mala calidad de sueño que reflejan los médicos residentes de las diferentes especialidades, y por lo tanto predisponen al padecimiento de trastornos del sueño, información respaldada por Aragón ${ }^{[2]}$ en donde hace referencia acerca del consumo de ansiolíticos como factor riesgo.
Los residentes que más han consumido sustancias para no dormir son los de las especialidades de ortopedia y pediatría por lo que para estas especialidades también son influyentes el consumo de sustancias para no dormir tanto en los trastornos como en la calidad del sueño, a como lo afirma Aragón ${ }^{[2]}$. (ver tabla 4)

Tabla 4. Consumo de sustancias para conciliar el sueño y sustancias para no dormir en los médicos residentes encuestados de las especialidades del HEODRA, Marzo a Octubre 2007

\begin{tabular}{ccc}
\hline Especialidad & $\begin{array}{c}\text { Consumo de sustancias } \\
\text { para conciliar el sueño }\end{array}$ & $\begin{array}{c}\text { Consumo de sustancias } \\
\text { para no dormir }\end{array}$
\end{tabular}

\begin{tabular}{lcccccccc} 
& \multicolumn{2}{c}{$\mathrm{Si}$} & \multicolumn{2}{c}{ No } & \multicolumn{2}{c}{$\mathrm{Si}$} & \multicolumn{2}{c}{ No } \\
\cline { 2 - 9 } & $\mathrm{N}^{0}$ & $\%$ & $\mathrm{~N}^{0}$ & $\%$ & $\mathrm{~N}^{\circ}$ & $\%$ & $\mathrm{~N}^{\circ}$ & $\%$ \\
\hline $\begin{array}{l}\text { Medicina } \\
\text { Interna }\end{array}$ & 2 & 20 & 8 & 80 & 7 & 70 & 3 & 30 \\
Ortopedia & 1 & 9 & 10 & 91 & 9 & 82 & 2 & 18 \\
Pediatría & 0 & 0 & 11 & 100 & 9 & 82 & 2 & 18 \\
Cirugía & 0 & 0 & 12 & 100 & 9 & 75 & 3 & 25 \\
Ginecología & 0 & 0 & 14 & 100 & 10 & 71 & 4 & 29 \\
\hline Total & 3 & & 55 & & 44 & & 14 & \\
\hline
\end{tabular}

Fuente Primaria

Figura 2. Perjudica de alguna manera a sus pacientes el no dormir del todo. HEODRA - período Marzo a Octubre del 2007

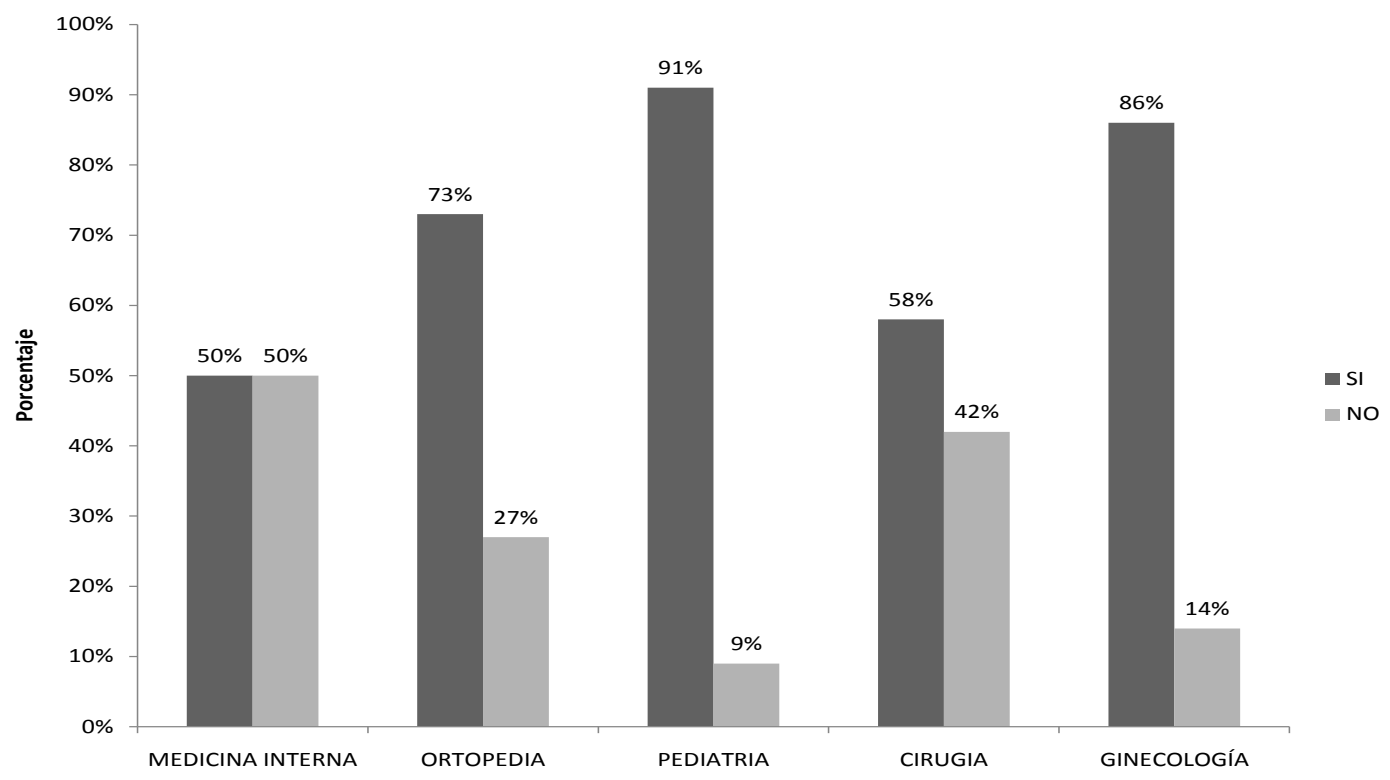

Fuente Primaria 
En las especialidades de pediatría, ginecología y ortopedia los residentes encuestados opinan que si perjudica de alguna manera a sus pacientes el no dormir del todo ya que se encuentran cansados, falta de concentración, no hay buena disponibilidad para atender a sus pacientes, susceptibilidad a cometer errores, en el ritmo de trabajo están mas lentos, muchas veces no se examina correctamente para un buen diagnóstico y se puede cometer iatrogenia en relación a las dosis, estas situaciones reflejan la situación de la práctica médica diaria y la importancia de mantener una buena calidad del sueño para brindar un servicio médico con calidad. (ver figura 2)

\section{CONCLUSIONES}

La calidad del sueño obtenida a través de la aplicación del Índice de calidad del sueño de Pittsburg refleja que todos los médicos residentes encuestados en el HEODRA tiene un puntaje mayor o igual a 5 puntos, siendo este el punto de corte para separar la buena calidad del sueño de la mala calidad.

La especialidad que refleja mala calidad con mayor puntaje es la de Medicina Interna, siendo los parámetros más afectados los de calidad subjetiva, eficiencia habitual, disfunción diurna y duración.

Los principales factores incidentes en un sueño de calidad en la población de estudio fueron los laborales, que incluyen el número de horas de trabajo, frecuencia de turnos, responsabilidades asignadas en cada turno y actividades académicas programadas para el día siguiente, en todas las especialidades hubo prevalencia de estos factores.

Otro de los factores que influyen en la calidad de sueño es el consumo de sustancias para mantenerse despiertos, principalmente el consumo de café, que es muy frecuente en los residentes de todas las especialidades.

El alcohol no interfiere como factor de riesgo para tener una mala calidad del sueño en los médicos residentes. Los principales trastornos del sueño que se determinaron con la realización de este estudio son: Insomnio de conciliación, insomnio de mantenimiento, insomnio tardío, sueño no reparador e insomnio transitorio, por lo que el padecimiento de estos trastornos dificultan la capacidad de concentración en la realización de sus actividades asignadas.

\section{REFERENCIAS BIBLIOGRÁFICAS}

1. ARAGÓN F., ALVARADO Q.(1997) Alteraciones del sueño en psiquiatría I. Febrero. ARAN, 13, № 2: 1027.

2. ARAGÓN F., ALVARADO Q. (1997) Alteraciones del sueño en psiquiatría I. Febrero. ARAN, 13, $\mathrm{N}^{\circ}$ 3: $13-30$.

3. SIERRA, JC, JIMÉNEZ, C. (2002) Calidad del sueño en estudiantes universitarios: importancia de la higiene del sueño. [monografía en internet]. Distrito Federal, México. Instituto nacional de psiquiatría Ramón de La Fuente. [consultado el 29 de abril del 2007] Disponible en: D:ICALIDADSUEÑOI Importanciadelahigiene del sueño.mht

4. HARRISON (2002) Principios de medicina interna. 15va Ed. México D.F. Editorial Mc Graw Hill interamericana.

5. VELLUTI, R. (1987) Esquema de la fisiología del sueño. Revista Médica Uruguay [internet] [consultado el 07 de mayo del 2007]; 3 [47-57].

6. MIRÓ E., MARTÍNEZ P. (2006) Influencia de la cantidad y la calidad subjetiva del sueño en la ansiedad y el estado de ánimo deprimido. [monografía en internet]. Distrito Federal, México. Salud Mental Instituto nacional de psiquiatría Ramón de La Fuente. Marzo - Abril [consultado el 29 de abril del 2007]. Disponible en: D:ICALIDAD SUEÑOICantiadadycalidadsubjetivadelsueño.mht 